\title{
Fluorescence Quenching by Colloidal Heavy Metals: Implications for Correlative Fluorescence and Electron Microscopy Studies
}

\author{
I.K. Kandela, D.A. Meyer, P.E. Oshel, E. Rosa-Molinar, and R. M. Albrecht \\ Department of Animal Sciences, University of Wisconsin, Madison, WI 53706
}

Colloidal heavy metals, such as gold, can be produced in a variety of sizes in the nanometer range. For labeling purposes the metal particles can be directly conjugated to various ligands or to antibodies via nonspecific hydrophobic interactions [1,2]. The heavy metal colloids are electron dense and have a distinct spherical shape and thus are very useful for both TEM and SEM applications [1,2,3]. They have a high extinction coefficient and produce a visible, inflated diffraction image and thus can be identified in several forms of light microscopy [1]. However, the use of the heavy metal colloids as light microscopic labels, particularly in correlative studies, is often problematic. Either the particle size or specimen geometry limits information attainable by interference based light systems. Enlarging smaller particles via silver enhancement may provide visibility in light microscopy, but the spatial resolution necessary for EM is lost. Thus, for correlative studies both in living and fixed systems, probes which combine fluorescence and colloidal heavy metal particles are useful. If colloidal particles of differing shapes or elemental compositions are used for high resolution co-localization studies, it would be advantageous to link a particular probe with a specific fluor such that co-localization at the light microscopic level (as for living cells) via fluorescence can be directly correlated with co-localization at the molecular level via EM [4]. Unfortunately, studies using UV-excited fluoresceinated identifier proteins such as FITC or rhodamine conjugated to colloidal metal particles result in quenching of the fluorescence. The degree of quenching seen with indirect labeling via fluorescent labels has not been well understood and is a possible alternative to direct conjugates. The use of mixtures of fluoresceinated identifier proteins and colloidal metal labeled proteins is also an alternative but competition between probes reduces the overall efficiency of labeling and hence detectability and spatial resolution for either probe.

In the current study we investigated the possibility of using primary colloidal gold-antibody conjugates with Alexa dyes as the fluorescent label. Direct Alexa-antibody-gold conjujgates and Alexa-antiantibody-antibody-gold were studied using scanning spectrofluoremetry and fluorescence light microscopy. In both systems the primary antibody is linked to the heavy metal particle which provides high spatial resolution and the best conditions for quantitative studies. Whether the fluorescent dye is directly conjugated to the primary antibody or ligand (most convenient) or to a second antibody does not significantly affect resolution in the light microscope because, in either case, the spatial resolution is well below that attainable in the light microscope.

Directly conjugating Alexa-protein to $18 \mathrm{~nm}$ and $5 \mathrm{~nm}$ colloidal gold particles resulted in a substantially decreased fluorescent signal with all three excitation wavelengths, 488, 546, and $594 \mathrm{~nm}$. Spectrofluorometric studies showed that the decrease in signal may reach $99 \%$ when compared to an equal amount of original fluorophore-protein not conjugated to gold. A range of excitation and emission wavelengths was checked to be certain there was no shift in excitation or emission frequencies. The intensity of the probes is insufficient to be observed with the fluorescence microscope via a reasonably sensitive Orca ER CCD camera (figs. 1a, 2a, 2b).

To determine if the quenching can be overcome by removing the fluorescent molecules farther from the gold $(10-20 \mathrm{~nm}$ as opposed to $1-6 \mathrm{~nm}$, rabbit $\mathrm{IgG}$ was conjugated to $18 \mathrm{~nm}$ colloidal gold and then 
labeled with goat Alexa-anti-IgG as a second label. In this configuration spectrofluorometry showed an $80 \%$ decrease in signal compared to free protein-fluorophore. While the quenching still exists, the signal is sufficient to be readily observed with the CCD camera in the fluorescent wide field LM and spinning disc confocal LM. Second antibody studies with smaller, $5 \mathrm{~nm}$, particles remain to be completed (figs. 1b, 2c, 2d).

References :

[1] R.M. Albrecht et. al., Immunochemistry, A Practical Approach, Oxford Univ. Press. Oxford (1993)151.

[2] R.M. Albrecht and D.A. Meyer. Micros.Microanal. 8 (Suppl.2) pp194-195, 2002.

[3] D.A. Meyer and R.M. Albrecht Proceedings 15th Int. Congress on Electron Microscopy, Vol 2, 53, 2002.

[4] E. Rosa-Molinar et.al., Micros. Microanal. 8 (Supp1.2) pp 132-133, 2002.

[5] Studies supported in part by NIH/NIGMS \# 63001.

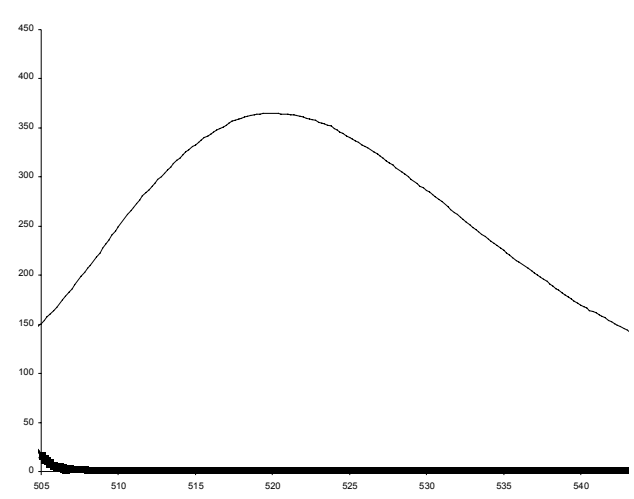

Figure 1a. _ Alexa 488-IgG

- Alexa 488-IgG-Gold

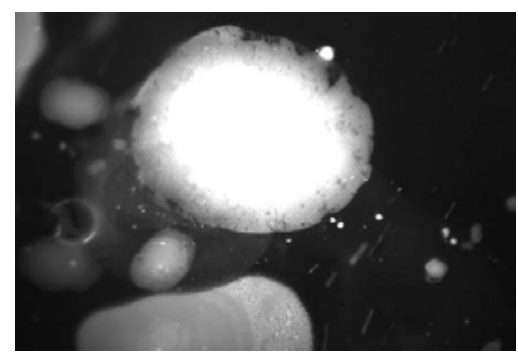

Figure 2a, Alexa488-IgG

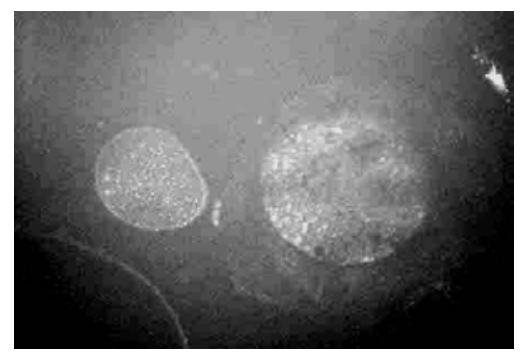

Figure 2c, Alexa488-antiIgG

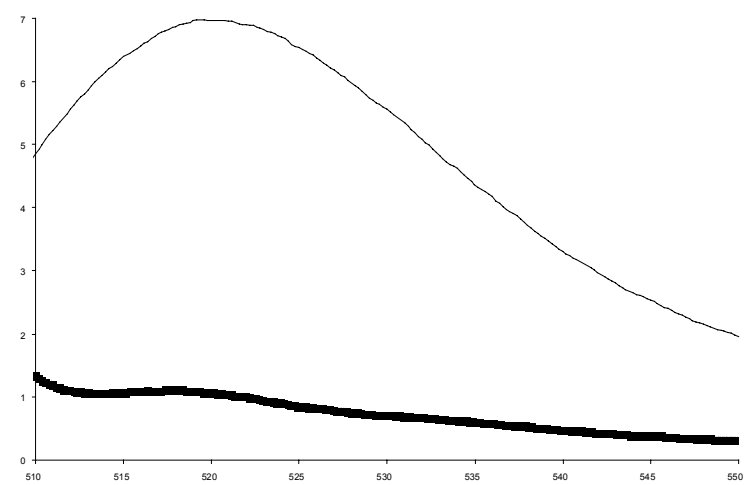

Figure $1 \mathrm{~b}$ - Alexa488-antiIgG - Alexa 488-antiIgG-IgG-Gold

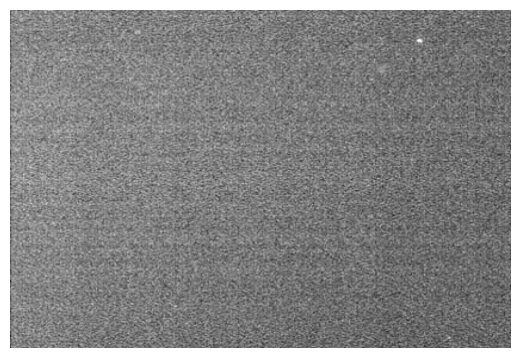

Figure 2b, Alexa 488-IgG-Gold

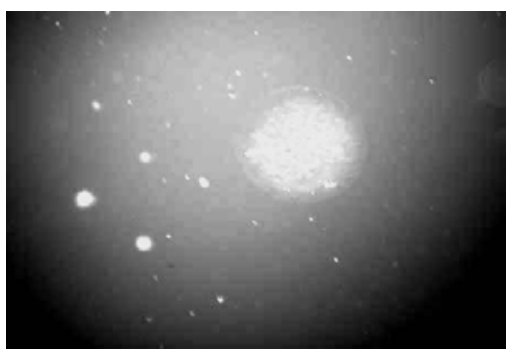

Figure 2d, Alexa488-antiIgGIgG-Gold 